\title{
Correction to: Medical Options for the Adjuvant Treatment and Management of Pediatric Melanoma
}

\author{
Haya S. Raef ${ }^{1}$ - Alison M. Friedmann ${ }^{2,3}$ • Elena B. Hawryluk $k^{2,4,5}$ \\ Published online: 25 May 2019 \\ C) Springer Nature Switzerland AG 2019

\section{Correction to: Paediatr Drugs (2019) 21(2):71-79 https://doi.org/10.1007/s40272-019-00326-w}

\section{Page 77, Table 1, 'Targeted Therapies'}

Study NCT01519323 (A study of vemurafenib in pediatric patients with stage IIIC or stage IV melanoma harbouring BRAFV600 mutations) was included in a table listing ongoing clinical trials of adjuvant therapies for pediatric melanoma (Table 1) in error. The study was in fact closed early due to low enrollment as correctly noted in section 4 of the article.

The original article can be found online at https://doi.org/10.1007/ s40272-019-00326-w.

Elena B. Hawryluk

ehawryluk@partners.org

1 Tufts University School of Medicine, Boston, MA, USA

2 School of Medicine, Harvard University, Boston, MA, USA

3 Department of Pediatrics, Massachusetts General Hospital, Boston, MA, USA

4 Department of Dermatology, Massachusetts General Hospital, 50 Staniford St., 2nd Floor, Boston, MA 02114, USA

5 Dermatology Program, Division of Immunology, Department of Medicine, Boston Children's Hospital, Boston, MA, USA 